\section{Homozygous deletion in Wilms tumours of a zinc-finger gene identified by chromosome jumping}

\author{
Manfred Gessler*, Annemarie Poustkat, \\ Webster Cavenee $\ddagger$, Rachael L. Neve\$, \\ Stuart H. Orkin\| \& Gail A. P. Bruns
}

Genetics Division, The Children's Hospital, and Department of Pediatrics, Harvard Medical School, Boston, Massachusetts 02115, USA

† German Cancer Research Center, Heidelberg, FRG

\$udwig Institute for Cancer Research, Montreal, Canada

$\$$ Department of Psychobiology, University of California-Irvine, Irvine,

California 92717, USA

|| Division of Hematology-Oncology, Children's Hospital and

Dana-Farber Cancer Institute, Department of Pediatrics,

Harvard Medical School, Howard Hughes Medical Institute, Boston,

Massachusetts 02115, USA

CYTOGENETIC analysis has identified chromosome $11 \mathrm{p} 13$ as the smallest overlap region for deletions found in individuals with WAGR syndrome, which includes Wilms tumour (a recessive childhood nephroblastoma), anirida, genito-urinary abnormalities and mental retardation' ${ }^{\prime}$. The underlying loci have since been resolved into an aniridia (AN2) locus at a telomeric position, and a locus of closely spaced genes or a single pleiotropic gene involved in genito-urinary tract abnormalities and Wilms tumour at a more centromeric position ${ }^{2-7}$. Pulsed-field gel analysis of the $11 \mathrm{p} 13$ region has revealed the presence of several putative $\mathrm{CpG}$ islands $\mathrm{s}^{8,9}$, structures which are frequently associated with the $5^{\prime}$ ends of

* Present address: Institut für Humangenetik, Philips-Universität, Marburg, FRG. expressed sequences, mainly housekeeping genes and some tissuespecific genes ${ }^{10}$. Starting from a $\mathrm{CpG}$ island, we have now isolated four neighbouring $\mathrm{CpG}$ islands, all within 650 kilobases (kb), by means of two consecutive bidirectional jumps in rare-cutting restriction-enzyme jumping libraries ${ }^{11}$. In two instances, flanking sequences were conserved in other species and RNA transcripts were identified. A complementary DNA clone isolated for one of them derives from an RNA highly expressed in fetal kidney, and is predicted to encode a Krüppel-like ${ }^{12}$ zinc-finger protein that is probably a transcription factor. The entire cDNA region is included in two partially overlapping homozygous deletions found in Wilms tumour DNA samples. Cloning of the breakpoints in one tumour revealed a deletion size of $170 \mathrm{~kb}$, one-third of which is covered by the cDNA. The expression pattern and sequence of this cDNA could point to an important role for its corresponding gene in the normal development of the renal system as well as in Wilms tumour.

We chose probe 282 , located close to the region implicated in Wilms tumour and genito-urinary tract abnormalities (WTGU region) and near two CpG islands, as a starting point. By chromosome walking, probe A282-EH2.6 containing sites for BssHII, SacII and other rare-cutting enzymes that are not methylated in genomic DNA was isolated. We expected that the 5.1-kb size of the corresponding EcoRI fragment would allow jumping in a Bss HII-EcoRI jumping library in both directions. Screening of $\sim 400,000$ clones yielded 11 strongly hybridizing colonies, with the inserts falling into two categories (LFC1 and LFT1), consistent with jumps in both directions (Fig. 1). As chromosome jumping is directional, the type of starting fragment indicated the direction of the jump, which we confirmed by mapping the new end fragments against somatic cell hybrid panels and by PFG analysis to provide correct placement on the pulsed-field (PFG) map (not shown).

To cross the CpG islands, the LFT1 and LFC1 jumping clones were used to isolate lapping $\lambda$ phage. In the case of LFT1-CP5, the large size of the telomeric Bss HII-EcoRI fragment $(\sim 10 \mathrm{~kb})$ precluded the use of the same BssHII-EcoRI jumping library for a consecutive jump. A second Bss HII jumping library, recut

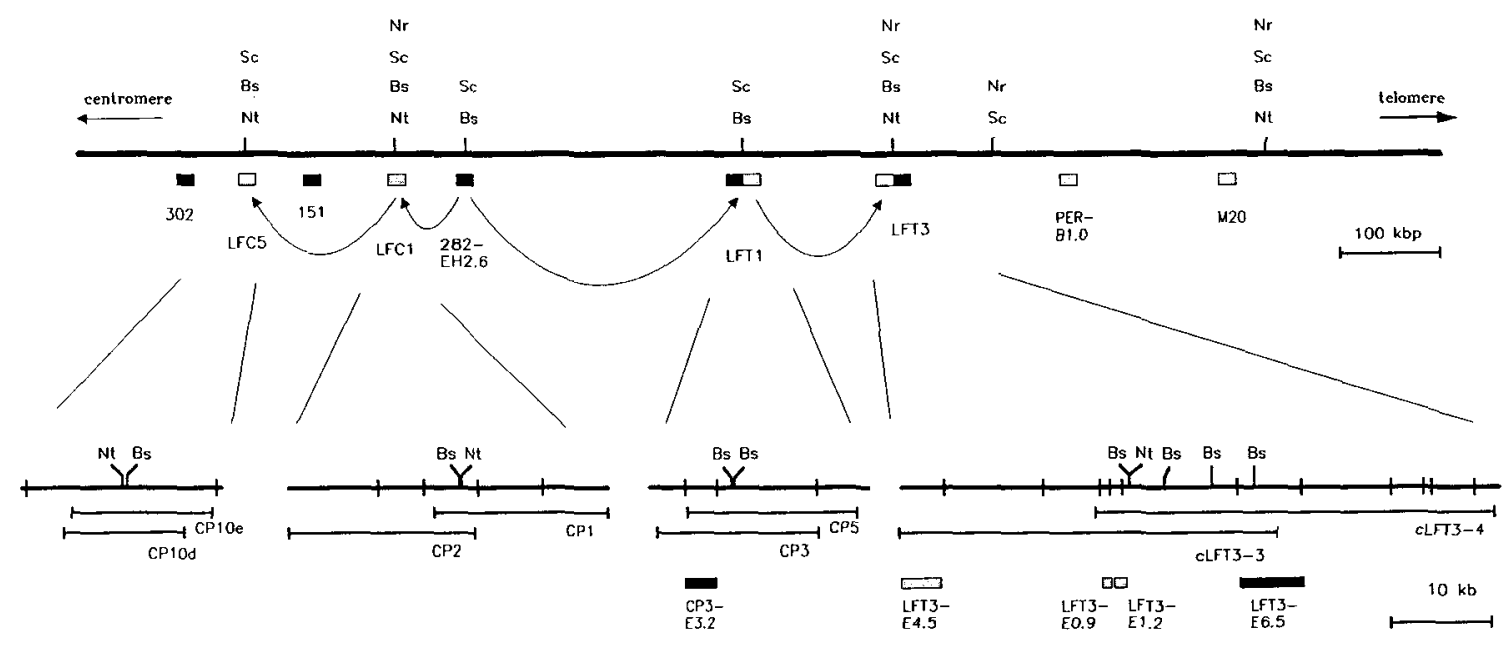

FIG. 1 The WT-GU region: Physical map and chromosome jumping. The PFG restriction map (top) from ref. 9 has been refined to include additional probes. The four directional jumps using probe A282-EH2.6 as a starting point are indicated with previously used random probes shown at their approximate positions. Phage or cosmid clones for the new CpG islands are drawn on a larger scale (bottom). Only two representative clones are shown in each case with their EcoRI restriction maps (vertical lines) and cutting sites for Notl and BssHHI. Shaded boxes depict the location of the probes used in Figs 2 and 4 ; conservation in rodent DNA is indicated by black boxes. Abbreviations: Nr, Nrul; Sc, Sacll; Bs, BssHIl; Nt, Notl.

METHODS. Probe A282-EH2.6 was isolated by two chromosome-walking steps in a Mbol partial digest library in vector EMBL3 from probe 282 (ref 5). The BssHII-EcoRI jumping library ${ }^{11}$ was plated as a lysogen onto MSI membranes and screened as previously described ${ }^{30}$. Eleven positive clones were picked and phage induced for re-screening. DNA was prepared from pure stocks and inserts released by $E_{c o R I}-S a l l$ cleavage. Fragments likely to represent the anticipated jump were subcloned into plasmid vectors and their position verified by mapping on somatic cell hybrid panels ${ }^{5}$ and comparison of the PFG pattern with that of the starting clone ${ }^{9}$. The new jump fragments were used to isolate longer phage clones from the EMBL3 library and fragments suitable for a consecutive jump were subcloned. The LFC5 and LFT3 jumps were isolated by screening 5,000 phage from a BssHII/BamHI-Hindlll jumping library with a 1.6-kb BssHIl-Hindlll fragment from CP1 and a 200-bp BssHII-Hindlll subclone of CP5, respectively. Correct inserts were again used to isolate overlapping EMBL3 or cosmid clones (Mbol partial digest human placental library in pWE15 provided by G. Evans). Restriction maps for these clones were derived from hybridizations of overlapping clones and by partial digest strategies. All subcloning and hybridization was done according to standard procedures ${ }^{31}$. 
FIG. 2 CpG island probes are conserved and contain expressed sequences. a, Subclones close to the $\mathrm{CpG}$ island map to the 11p13-11p14 region and cross-hybridize with rodent DNA (arrows). $b$ These probes also detect RNA transcripts in fetal RNA (1, kidney; 2, heart; 3 , thymus; 4 , liver; 5 , lung; 6, brain). The CP3E3.2 transcript is expressed in all tissues. Expression of the LFT3-associated transcript was only observed in fetal kidney.

METHODS. a, Somatic cell hybrid panels (EcoRIdigested human DNA (1), hamster DNA (2), human hamster hybrid DNA containing a normal chromosome 11 (3) or WAGR deletion chromosomes $(4,5)$ and human mouse hybrid DNA (6) containing 11pter-q23) described in ref. 5 were hybridized and washed under normal stringency. Bands in hamster (ianes 2-5) and mouse (lane 6) DNA are marked by arrows. b. Fetal RNAs (20-22 weeks) were prepared by the GuSCN-CsCl 2 method, $10 \mu \mathrm{g}$ each separated on a $1.1 \%$ agarose-formaldehyde gel and transferred to Genatran membrane. Hybridization was performed under standard conditions $\left(50 \%\right.$ formamide, $\left.5 \times \mathrm{SSC}, 42^{\circ} \mathrm{C}\right)$ with washing in $0.5 \times$ SSC, $0.5 \%$ SDS at $55^{\circ} \mathrm{C}$ followed by overnight exposure. A 600-base pair (bp) AvallHincll fragment of CP3E3.2 and the 1.6-kb EcoRI fragment of the LK1 cDNA clone were used as probes. Size estimates were derived from the positions of ribosomal RNA and a BRL RNA ladder.
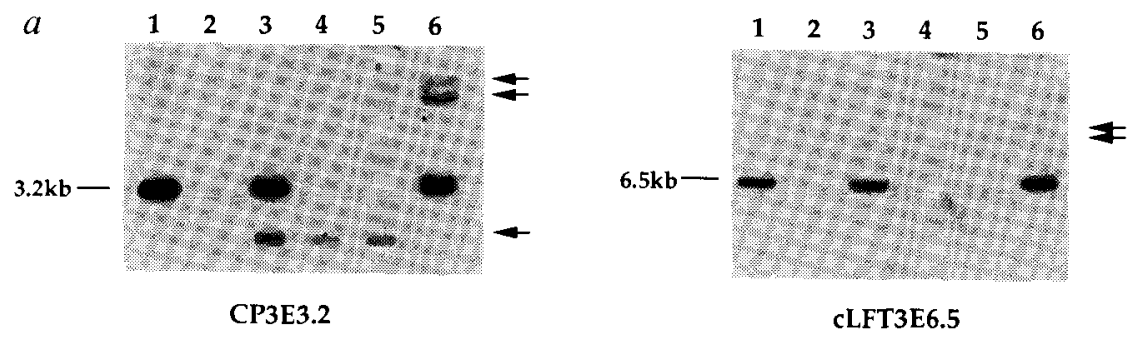

$b$

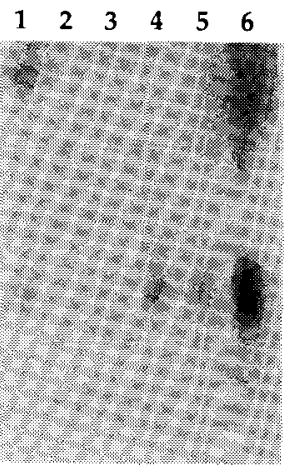

CP3E3.2

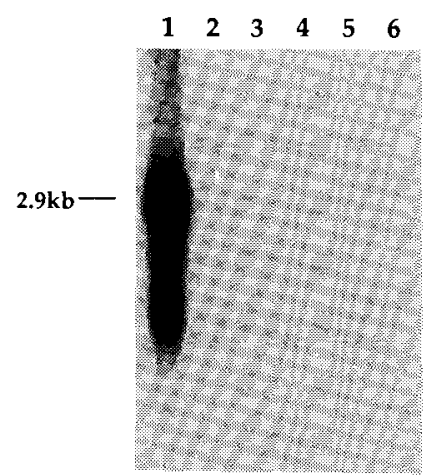

LK1E1.6 with the enzymes Hind III and BamHI instead of Eco RI, circumvented this problem ${ }^{13}$. Two identical clones (LFT3) from this library mapped to the correct chromosomal position (Fig. 1). Screening of a human cosmid library yielded four overlapping clones, spanning $\sim 60 \mathrm{~kb}$. Hybridization with probes from these cosmid clones confirmed their expected position on the PFG map. We used the Bss HII/BamHI-HindIII jumping library to isolate another jump clone (LFC5), centromeric to LFC1 (Fig. 1). Again, overlapping $\lambda$ clones (CP10) were isolated to encompass the entire $\mathrm{CpG}$ island. We therefore isolated four new $\mathrm{CpG}$ islands together with their flanking genomic regions.

Several genomic fragments on each side of every $\mathrm{CpG}$ island were assayed for cross-hybdrization with rodent DNA on somatic cell hybrid panels under high stringency. At two of the four $\mathrm{CpG}$ islands we detected conserved fragments (Fig. 2a). On northern blots, a subclone of CP3E3.2 detected a transcript of $\sim 1 \mathrm{~kb}$ (Fig. $2 b$ ) expressed in all fetal tissues examined, consistent with the characteristics of a housekeeping gene. With the 6.5-kb EcoRI fragment of cLFT-6, a hybridization signal was seen only with fetal kidney RNA (Fig. $2 b$ ) among the tissues examined. We investigated this transcript further, because it maps in the WT-GU subregion of the WAGR locus.

A total of 15 cDNA clones for the LFT3-associated transcript were isolated from a human fetal kidney cDNA library. The longest clone ( $3 \mathrm{~kb}$ ), designated LK15 (Fig. $3 a$ ), is similar in size to the RNA transcript, estimated to be $2.9 \mathrm{~kb}$. The transcript probably originates from the CpG island, as the $5^{\prime}$ end of LK15 maps within $4 \mathrm{~kb}$ of the island. Analysis of genomic DNA and overlapping cosmid clones indicated that the corresponding locus is spread over $\sim 60 \mathrm{~kb}$.

Sequence analysis of the cDNA clones revealed a combined length of 3,020 nucleotides with a single long open reading frame of 1,725 nucleotides starting at the 5' end of LK15 (Fig. $3 b$ ). The first ATG codons (positions 381 and 759) in the cDNA sequence do not fit the consensus sequence for initiation codons ${ }^{14}$. This observation, taken together with that of an open reading frame extending to the end of the clone, implies that the translation start site of the messenger RNA has not yet been cloned.

The predicted protein contains four tandemly repeated sequence motifs (Fig. $3 c$ ) related to the zinc-fingers of the product of Krüppel, a Drosophila segmentation gene ${ }^{12}$. By sequence comparison, the murine Krox-20-encoded polypeptide and its human homolog EGR2 were most similar to that encoded by clone LK15 with $63.1 \%$ amino-acid identity in the zinc-finger domain ${ }^{15,16}$. Krox-20 is an early growth response gene activated during G0-G1 transition in cultured cells ${ }^{15}$.

Another feature of the translated cDNA sequence is the unusually high proline content, with two regions between amino acids 5 and 46 and 153 and 209 containing 33\% proline. The latter region includes a stretch of nine prolines, reminiscent of the cluster of seven prolines near the $\mathrm{N}$ terminus of the protein encoded by $\mathrm{Krox}-20^{15}$. Proline-rich regions have also been found in several other putative DNA-binding proteins ${ }^{17}$, including the Krüppel gene product, and could represent transcriptional activation domains as described for the CTF/NF-1 family of closely related polypeptides ${ }^{17}$. From these structural features, it is reasonable to infer that the product of the LK15 cDNA clone is a DNA-binding protein that could be a transcriptional regulator.

We also tested all new $\mathrm{CpG}$ islands against a panel of DNAs from 65 Wilms tumours to detect possible deletions or rearrangements. The centromeric clones as well as LFT1 detected no homozygous deletions on Southern blots. But, the telomeric LFT3 probe identified homozygous deletions in two tumours (Fig. 4a,b). Both deletions start between the LFT1 and LFT3 $\mathrm{CpG}$ islands and encompass all fragments detected by the LK15 cDNA clone, but do not include the more distal probe M20 (Fig. 4c). The proximal breakpoint in tumour PER is located only $\sim 2 \mathrm{~kb}$ centromeric to the CpG island on the $500-\mathrm{kb}$ Not I fragment. We cloned the rearranged EcoRI fragment of $10 \mathrm{~kb}$ and mapped the PER B1.0 subclone to the 375-kb Not I fragment between the LK15 cDNA and probe M20. More detailed mapping (not shown) revealed a deletion size of $\sim 170 \mathrm{~kb}$ with both alleles having apparently identical deletions.

The homozygous deletion in Wilms tumour 8A terminates proximal to that in PER, as is also the case for the WiT-13 deletion that includes the CpG island and part of the $375-\mathrm{kb}$ Not I fragment ${ }^{18,19}$. The distal end of the Wilms tumour $8 \mathrm{~A}$ deletion has not been identified but must be located in the PER deletion. The two deletions therefore overlap by $<170 \mathrm{~kb}$, with $60 \mathrm{~kb}$ covered by the LK 15 locus (Fig. $4 c$ ). It seems quite feasible to isolate the remaining region of overlap of both homozygous deletions to determine whether other genes adjacent to LK15 
$a$

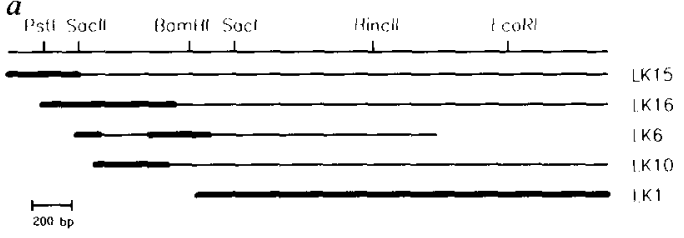

FIG. 3 Sequence of the LK CDNA clones and the zinc-finger motif. a Restriction map of CDNA isolates and sequencing strategy. The clones used for sequence analysis are shown with sequenced portions represented by thick lines. $b$, Nucleotide sequence of the LK cDNAs with the deduced amino-acid sequence (single-letter code) for the open reading frame. The proline-rich regions are located in the $\mathrm{N}$-terminal half of the sequence. The zinc-finger domain extends from nucleotides 1,329 to 1,694 , followed by a translation termination codon at position 1,728. The 3 ' untranslated region contains a simple repeat element (G-T) (nucleotides $2,751-2,793$ ) and a polyadenylation signal A-T-T-A-A-A $(2,985-2,990)$, followed by a 16-nucleotide poly(A) tract in LK1. C. Deduced amino-acid sequence (single-letter code) of the putative LK-associated zinc fingers, consensus sequence and comparison with the similar Krox-20-EGR2 consensus sequence ${ }^{15.16}$. Capital letters indicate strict conservation, other conserved amino acids are printed in lower-case letters. Boxes indicate the His-Cys link $(\mathrm{H}-\mathrm{C})^{32}$ and amino acids strictly conserved in the Drosophila Krüppel gene product.

METHODS. A CDNA library was prepared from $3 \mu \mathrm{g}$ fetal kidney poly(A) RNA (20-22 weeks) using the Invitrogen librarian kit. After size selection of the CDNA $(>1.5 \mathrm{~kb})$ ligation into $\lambda$ gt10 and packaging using Gigapack gold (Stratagene) $2 \times 10^{6}$ clones were obtained. Screening of part of the library with a 3-kb LFT3E6.5 subfragment, competed with excess sheared human placental DNA, led to the isolation of six cDNA clones. Re-screening with these cDNA clones resulted in isolation of a total of 15 clones with consistent restriction maps. The longest clone (LK15) is $3 \mathrm{~kb}$. The entire CDNA maps to the 11013 region in the WT PER-8A deletion area (Fig. 4). Sequence analysis was performed on plasmid subclones using the USB Sequenase kit. Both strands were sequenced. Compressions in the $5^{\prime} \mathrm{GC}$-rich regions were resolved by substituting deaza-GTP for GTP. The UWGCG program package was employed for sequence editing and comparison. The zinc-finger domain is underlined and the polyadenylation signal is overlined. $b$ TCAAGGCAGCGCCCACACCCGGGGGCTCTCCGCAACCCGACCGCCTGTCCGCTCCCCCACTTCCCGCCCTCC S R R R P P I P P G A $\begin{array}{llllllllllllllllllllllll}L & P & P & T & \text { I } & S & P & T & \text { A } & P & P & R & A & G & T & A & A & Q & A & P & G & P & R & R\end{array}$ CTCCTCGCCGCGATCCTGGACTTCCTCTTGCTGCAGGACCCGGCTTCCACGTGTGTCCCGGAGCCGGCGTCT

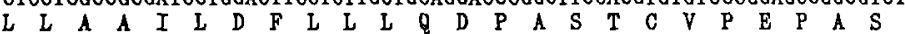
CAGCACACCTCCGCTCCGGGCTGGTCCCTACAGCAGCCAGAGCAGCAGGGAGTCCGGGACCGGGGCGGC

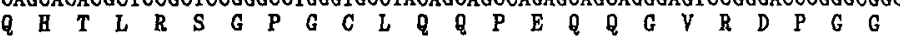
ATCTGGGCCAAGTTAGGCGCCGCCGAGGCCAGCGCTGAACGTCTCCAGGGCCGGAGGAGCCGCGGGGCGTCO

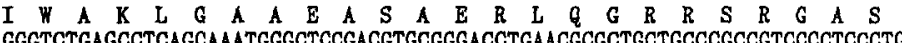
GGGTCIGAGCCTCAGCAAATGGGCTCCGACGTGCGGGACCTGAACGCGCTGCTGCCCGCCGTCCCCTCCCTC

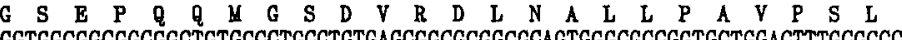
G G G G G C A L P CCGGGCGCTTCGGCTTACGGGTCGTTGGGCGGCCCCGCGCCCCCACCGGCTCCGCCGCCACCCCCGCCGCCO

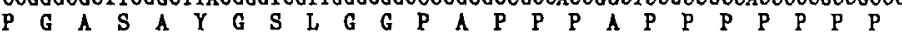
CCGCCTCACTCCTTCATCAMCAGGACCCGGCTCOCOCCCCGAGCCGACGAGCACAGTCCCTGAGC $P$ P H S F I K Q E P S W G G A E P H E E Q C L S GCCTTCACTGTCCACTTTTCCGGCCAGTTCACTGGCACAGCCGGAGCCTGTCGCTACGGGCCCTTCGGTCCT $\begin{array}{llllllllllllllllllllllll}A & F & T & V & A & F & S & G & Q & F & T & G & T & A & G & A & C & R & Y & G & P & F & G & P\end{array}$ CCTCCGCCCAGCCAGGCGTCATCCGGCCAGGCCAGGATGTTTCCAACGCECCCIACCTGCCCAGCTGCCTC

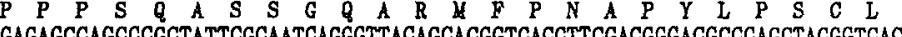

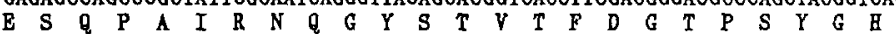
ACGCCCTCGCACCATGCGGCGCAGTTCCCCAACCACTCATTCAAGCATGAGGATCCCATGGGCCAGCAGGGO $T$ P S H H A A Q F P N B S F K H E D P M G Q Q G TCGCTGGGTGAGCAGCAGTACTCGTGCGCCCCGGTCTATGGCTGCCACACCCCCACCGACAGCTGCACO

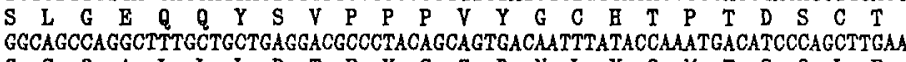

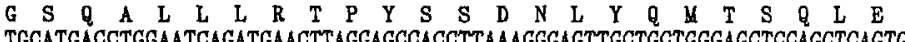
C M T W N \& M N L G A T L K G V A A G S S S S S V

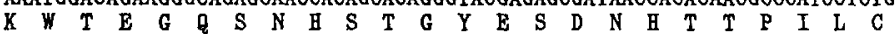
GGAGCCCAATACAGAATACACACGCACGGTGTCTTCAGAGGCATTCAGGATGTGCGACGTGTGCCTGGAGTA

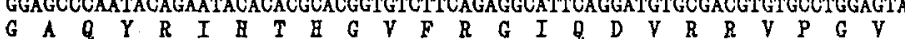
GCCCCGACTCTTGTACGGTCGGCATCTGAGACCAGTGAGAACGCCCCTTCATGTGTGCTTACCCAGGCTGO GCCCGACTCTTGTACGGTCGGCATCTGAGACCAGTGAGAAACGCCCCTICATGTGTGCTTACCCAGGCTGC A
AATAAGAGATATTTTAAGCTGTCCCACTTACAGATGCACAGCAGGAAGCACACTGGTGAGAACCATACCAG

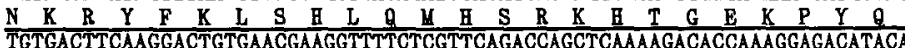

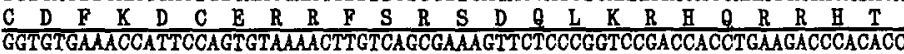

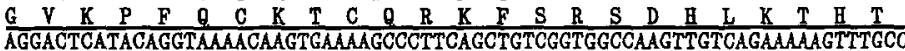

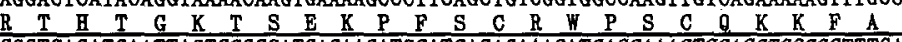

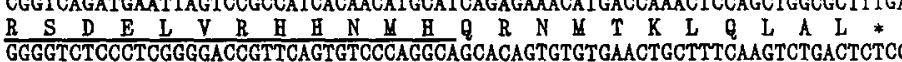
GGGGTCTCCCTCGGGGACCGTTCAGTGTCCCAGGCAGCACAGTGTGTGAACTGCTTTCAAGTCTGACTCTCC ACTCCTCCTCACTAAAAAGGAAACTTCAGTTGATCTTCTTCATCCAACTTCCAAGACAAGATACCGGIGCT CTGGAAACTACCAGGTGTGCCTGGAAGAGTTGGTCTCTGCCCTGCCTACTTITAGT GACTCACAGGCCTO GAGAAGCAGCTAACAATGTCTGGTTAGTTAAAACCCATTGCCATTTGGTCTGGATTTTCTACTGTAAGAA AGCCATAGCTGATCATGTCCCCCTGACCCTTCCCTTCTTTTTTTATGCTCGTTTTCGCTGGGGATGGAATT TTGTACCATTTTCTATCATGGAATATTTATAGGCCAGGGCATGTGTATGTGTCTGCTAATGTAAACTTTGTO ATGGITTCCATTTACTAICAGCAACAGCAAGAAATAAATCAGAGAGCAAGGCATCGGGGGTGATTCTTGTCT ACATTCCCGAGGTCAGCCAGGCTGCTAACCTGGAAAGCAGGATGTAGTTCTGCCAGGCAACTTTAAAGGT AACATTCCCGAGGTCAGCCAGGCTGCTAACCTGGAAAGCAGGATGTAGTTCTGCCAGGCAACTTTTAAAGCT CATGCATTTCAAGCAGCTGAAGAAAGAATCAGAACTAACCAGTACCTCTGTATAGAAATCTAAAAGAATTT ACCATTCAGTTAATTCAATGTGAACACTGGCACACTGCTCTTAAGAAACTATGAAGATCTGAGATITTTTTG TGTATGTTTTTGACTCTTTTGAGTGGTAATCATATGTGTCTTTATAGATGTACATACCTCCTTGCACAAATG GAGGGGAATTCATTTTCATCACTGGGACTGTCCTTAGTGTATA AAAACCATGCTGGTATATGGCTTCAAGTI GTAAAATTGAAGTGACTTTAAAAGAAAATAGGGGATGGTCCAGGATCTCCACTGATAAGACTGTTTTTAAG TAACTTAAGGACCTTTGGGTCTACAGGTATATGTGAAAAAAATGAGACTTACTGGGTGAGGAAATCCATTGT TTAAAGATGGTCGTGTGTGTGTGTGTGTGTGTGTGTGTGTTGTGTTGTGTTTIGTTTITTAAGGGAGGGAAT TTATTATTTACCGTTGCTTGAAATTACTGTGTAAATATATGTCTGATAATGATTTGCTCTTTGACAACTAAA

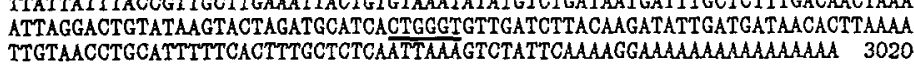

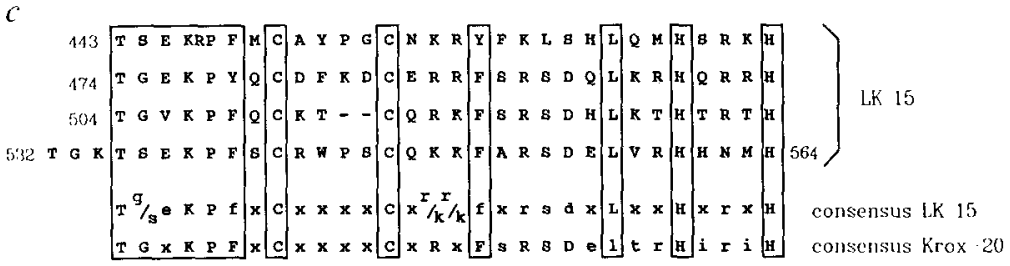

play a part in the development of the genito-urinary system or in Wilms tumour.

The low incidence of homozygous deletions that are detected by our probes, which can be at most $50 \mathrm{~kb}$ away from a Wilms tumour locus in this region, and the presence of an apparently normal-sized transcript for LK15 in 12 tumours (data not shown), contrasts with the frequent deletions and internal rearrangements of $R b-1$, another tumour suppressor gene, seen in retinoblastomas and other tumours ${ }^{20-24}$. It is possible that the presence of another important gene in this region favours moresubtle alterations in these tumours, or loci at other sites in the genome $\mathrm{e}^{25-29}$ could have a larger role in sporadic Wilms tumours than previously believed.

The expression pattern of the LK15 transcript indicates that its corresponding gene could be involved in normal kidney development, especially because the genito-urinary anomaly component of the WAGR syndrome maps to this genomic region $^{6,7}$. The presence of the Krüppel-like ${ }^{12}$ zinc-finger domain in the LK15 cDNA and its potential function as a regulatory protein is consistent with this model.

Our identification of this zinc-finger gene demonstrates the power of the combined approach of mapping $\mathrm{CpG}$ islands by 
FIG. 4 The LFT3-LK1 region is homozygously deleted in two Wilms tumours. a, Probe LFT3E4.5 detects a band in DNA from Wilms tumour PER whereas Probe EO.9 detects a fragment of altered size, and the adjacent probe E1.2 is homozygously deleted. All bands detected by the LK1 CDNA are also deleted. The PER B1.0 probe, isolated from the other side of the cloned deletion junction, detects the same rearranged band in PER DNA as probe E0.9. $b$, in Wilms tumour $8 A$, the proximal boundary of the homozygous deletion region is located between probes CP5E3.2 and probe LFT3E4.5. The entire LK1 CDNA region including the $3^{\prime}$ end (not shown) is deleted in this DNA. The PER B1.0 probe, however, is not included in the homozygous deletion. The extremely weak $1.8 \mathrm{~kb}$ band in both PER and 8A that is seen with LK1E1.6 is probably due to a small percentage of normal cells in the tumour samples. $c$, Schematic rep resentation of the extent of homozygous deletions in tumours PER and 8A. Dashed lines indicate uncertainty of the exact breakpoint location in $8 \mathrm{~A}$ The extent of the LK15 locus is derived from the analysis of additional overlapping cosmid clones (not shown). The size of the PER deletion was measured by PFG mapping studies not shown here. METHODS. DNA $(4 \mu \mathrm{g})$ from tumours and norma control DNA (C) were digested with EcoRI (or $B a m H I$ for probe PER B1.0 in b), and filters were prepared and hybridized as described ${ }^{5}$. Before using filters again they were stripped in $10^{-3} \mathrm{M}$ Tris-HCL, pH 8,10 $10^{-4} \mathrm{M}$ disodium EDTA, 0.1\% SDS at $75^{\circ} \mathrm{C}$. To clone the rearranged fragment in PER, EcoRl-digested tumour DNA was ligated into EMBL4 and packaged using Gigapack gold (Stragagene). Screening with LFT3EO.9 gave eight independent isolates. After restriction mapping of the $10-\mathrm{kb}$ deletion junction, suitable fragments were subcloned into plasmid vectors. The PER B1.0 clone correctly maps to chromosome 11p13 and is located $\sim 170 \mathrm{~kb}$ telomeric to the LFT3 CPG island as determined by PFG analysis. Abbreviations as in Fig. 1.

PFG analysis and subsequent cloning by consecutive jumps from one island to the next using rare-cutting restriction-enzyme jumping libraries. Molecular probes for island-associated genes

Received 2 November 1989; accepted 9 January 1990.

1. Francke, U., Holmes, L. B.. Atkins, L. \& Riccardi, V. M. Cytogenet. Cell Genet. 24, 185-192 (1979). 2. Turleau, C. et al. Hum. Genet. 67, 455-456 (1984)

2. Turleau, C. et al. Hum. Genet. 67. 455-456 (1984)

4. Glaser, T. et al. Nature $321,882-887$ (1986). $34,535-5359$ (1987)

4. Porteous. D. et al. Proc. natn. Acad. Sci. U.S.A. 84, 5355-5359

5. Gessler, M. et al. Am. J. hum. Genet. 44, 486-495 (1989).
6. Gessler, M. \& Bruns, G. A. P. Cytogenet. Cell Genet. 51, 1003 (1989)

7. van Heyningen, $v$. et al. Cytogenet. Cell Genet. 51, 1095 (1989)

8. Compton, D. A. et al. Cell 55, 827-836 (1988)

9. Gesster, M. \& Bruns, G. A. P. Genomics 5, 43-55 (1989).

10. Bird. A. P. Nature 321, 209-213 (1986).

11. Poustka, A., Pohl, T. M., Barlow, D. P. \& Frischauf, A.-M. Nature 325, 353-355 (1987)

12. Rosenterg, $U$. et at. Nature 319, 336-339 (1986)

13. Poustka, A. \& Lehrach, H. Genet. Engin. Vol. 10 (ed. Setlow, J. K.) 169-195 (Plenum Press. New York and London, 1988).

14. Kozak. M. Nucleic. Acids Res. 15, 8125-8132 (1987).

15. Chavier, P. et al. EMBO \&. 7. 29-35 (1988).

15. Chavier, P. et al. EMBO J. 7. 29-35 (1988).

16. Joseph, L. J. et al. Proc. natn. Acad. Sci, U.S.A. 85, 7164-7168 (1988).

17. Mermod, N., ONeill, E. A., Kelly, T. J \& TJan, R. Cell 58, 741-753 (1989). (1989).

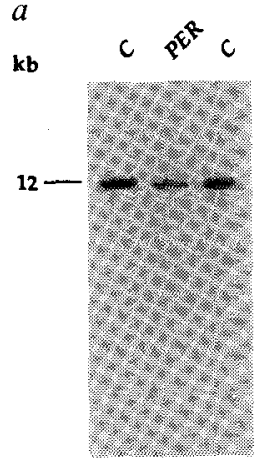

cLFT3E4.5
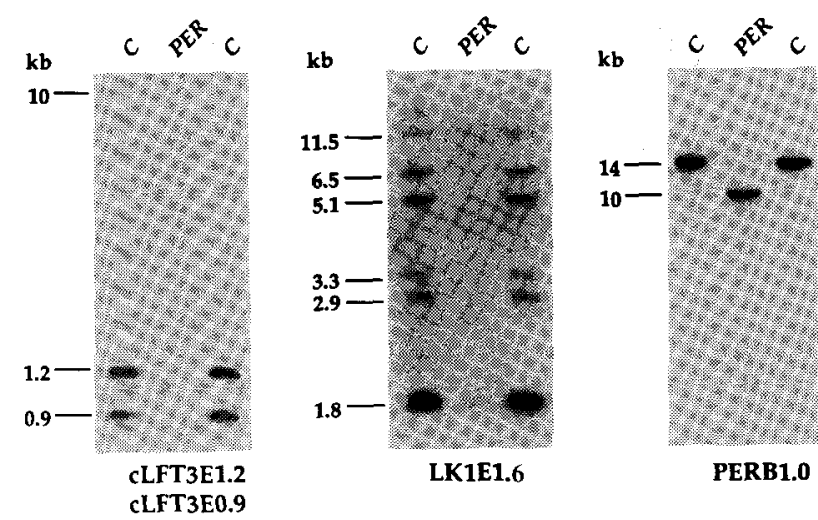

$b$

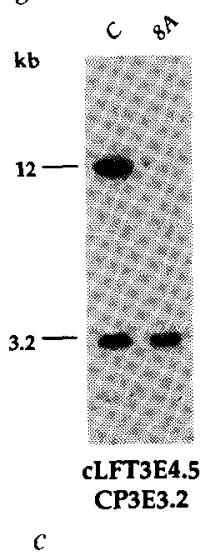

c 8

$\mathbf{k b}$

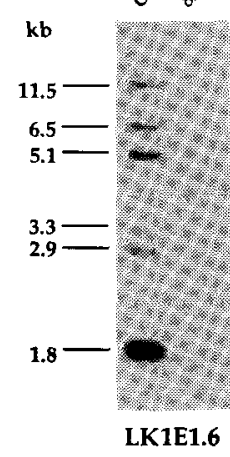

$c$ at

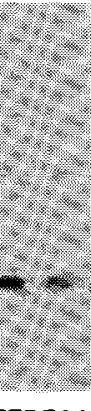

PERB1.0

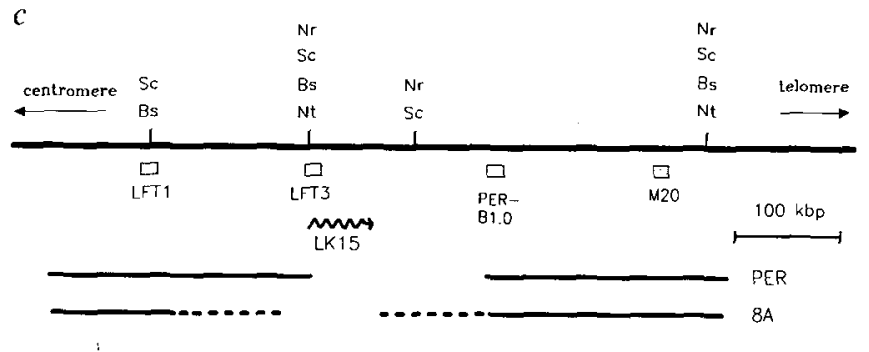

should access a significant fraction of expressed sequences, and, especially in chromosomal regions rich in $\mathrm{CpG}$ islands, provide a framework for a gene map.

19. Call, K. et al. Cytogenet. Cell Genet. 51, 974 (1989).

20. Friend, S. H. et al. Nature 323, 643-646 (1986)

21. Fung, Y.K. T. et al. Science 236, 1657-1661 (1987)

22. Lee. W. H. et al. Science 235, 1394-1399 (1987)

23. Goddard, A. D. et al Molec. cell. Biol, 8, 2082-2088 (1988).

24. Harbour, J. W. et al. Science 241, 353-357 (1988).

25. Grundy, $P$. et al. Nature 336, 374-376 (1988).

26. Huff, V. et al. Nature 336, 377-378 (1988).

27. Koufos. A. et al. Am. \&. hum Genet 44, 711-719 (1989).

27. Koufos. A. et al. Am. J. hum. Genet. 44, 711-719 (1989).
28. Henry, I. et al. Proc. natn. Acad. Sci. U.S.A. 86, 3247-3251 (1989).

29 Reeve, A. E Sih. S. A.. Raizis, A. M. \& Feinberg. A. P. Molec. cell. Biol 9, 1799-1803 (1989)

30. Herrmann, B. G., Barlow, D. P. \& Lehrach, H. Cell 48, $813-825$ (1987).
31. Maniatis, T., Fritsch, E. F. \& Sambrook, J. Molecular Cloning (Cold Spring Harbor Laboratory, Cold

11. Maniatis, T., Fritsch, E. F. \& Sambrook, J. Molecular Cloning (Cold Spring Harbor Laboratory, Cold Spring Harbor, New York, 1982)

32. Schuh, R. et al. Cell 47, 1025-1032 (1986).

ACKNOWLEDGEMENTS. We thank S. Sallan, F. Li, J. Mandell. C. Peters and S. Ruyle for providing Wilms tumour DNA samples This work was supported by the NIH (G.A.P.B. and R.L.N.) S.HO. is an Investigator of the Howard Hughes Medical Institute. A.P. is partly supported b; a Hereditary Disease Foundation grant to Hans Lehrach. M.G. is recipient of a postdoctoral fellowship from the Deutsche Forschungsgemeinschaft. 